Article

\title{
Polydopamine-Inspired Surface Modification of Polypropylene Hernia Mesh Devices via Cold Oxygen Plasma: Antibacterial and Drug Release Properties
}

\author{
Xiakeer Saitaer ${ }^{1,2}$, Noor Sanbhal ${ }^{2,3}$, Yansha Qiao ${ }^{2}$, Yan Li ${ }^{2, *} \mathbb{0}$, Jing Gao ${ }^{2}$, Gaetan Brochu ${ }^{4}$, \\ Robert Guidoin ${ }^{2,4}$, Awais Khatri ${ }^{3}$ and Lu Wang ${ }^{2, *}$ \\ 1 College of Textiles and Fashion, Xinjiang University, 666 Sheng Li Road, Tian Shan, Wulumuqi 830046, \\ China; xaker2@163.com \\ 2 Key Laboratory of Textile Science and Technology of Ministry of Education, Key Laboratory of Biomedical \\ Textile Materials and Technology in Textile Industry, College of Textiles, Donghua University, 2999 North \\ Renmin Road, Songjiang, Shanghai 201620, China; noor.sanbhal@faculty.muet.edu.pk (N.S.); \\ qiaoys233@163.com (Y.Q.); gao2001jing@dhu.edu.cn (J.G.); robert.guidoin@fmed.ulaval.ca (R.G.) \\ 3 Department of Textile Engineering, Mehran University of Engineering and Technology, Jamshoro, \\ Sindh 76062, Pakistan; awais.khatri@faculty.muet.edu.pk \\ 4 Department of Surgery, University of Laval, Quebec, QC G1V 0A6, Canada; gaetan.brochu@chg.ulaval.ca \\ * Correspondence: yanli@dhu.edu.cn (Y.L.); wanglu@dhu.edu.cn (L.W.); Tel./Fax: +86-21-67792637 (L.W.)
}

Received: 20 December 2018; Accepted: 22 February 2019; Published: 1 March 2019

\begin{abstract}
Mesh infection is a major complication of hernia surgery after polypropylene (PP) mesh implantation. Modifying the PP mesh with antibacterial drugs is an effective way to reduce the chance of infection, but the hydrophobic characteristic of PP fibers has obstructed the drug adhesion. Therefore, to prepare antimicrobial PP mesh with a stable drug coating layer and to slow the drug release property during the hernia repair process has a great practical meaning. In this work, PP meshes were coated by bio-inspired polydopamine (PDA), which can load and release levofloxacin. PP meshes were activated with cold oxygen plasma and then plasma activated PP fibers were coated with PDA. The PDA coated meshes were further soaked in levofloxacin. The levofloxacin loaded PP meshes demonstrate excellent antimicrobial properties for 6 days and the drug release has lasted for at least $24 \mathrm{~h}$. Moreover, a control PP mesh sample without plasma treatment was also prepared, after coating with PDA and loading levofloxacin. The antimicrobial property was sustained only for two days. The maximum inhibition zone of PDA coated meshes with and without plasma treatment was 12.5 and $9 \mathrm{~mm}$, respectively. On all accounts, the modification strategy can facilely lead to long-term property of infection prevention.
\end{abstract}

Keywords: polypropylene; hernia meshes; antibacterial; drug release; polydopamine

\section{Introduction}

An abdominal wall hernia operation is the repositioning of the hernia contents within the abdominal cavity using sutures or mesh materials. Abdominal closure or reinforcement of hernia defects has been performed with numerous mesh materials. Indeed, mesh implantation for hernia repair has reduced hernia recurrence rate, compared to suturing techniques [1]. Synthetic mesh materials have been successful for hernia repair since the last decade. Nevertheless, among all these materials, polypropylene (PP) mesh has been considered as one of the best materials for repairing hernias, owing to its inherent characteristics including that it is inert, hydrophobic, has a strong mechanical property, and is lightweight [2-4]. However, a significant complication of hernia repair is PP mesh induced infection [5,6]. In addition, PP is a hydrophobic polymer which does not absorb 
drugs during pre-operative prophylaxis [7,8], thereby making the drug coating, to prevent infection, infeasible for PP mesh. Mesh infection can be a serious problem if not solved in the initial stages of mesh implantation. After infection, it is difficult to cure the infected surgical area because heavy antibiotic doses to the body of the patient may cause side effects. Furthermore, the recurrence of the hernia may cause the removal of the hernia mesh, which can be costly in terms of the wealth and health of patients $[9,10]$. Therefore, creating an active surface of PP mesh and providing the binding sites for drug loading during mesh implantation are the key factors to reduce PP hernia mesh infection [11]. Different kinds of surface modifications of PP meshes have been performed to incorporate different antibiotics for slow drug release $[12,13]$. Amongst those modification protocols, plasma treatment is considered a crucial step to modify the hydrophobic surfaces of PP fibers [14,15], to incorporate the functional groups, such as hydroxyl or carboxyl, on the surfaces of PP fibers [16], and those functional groups were further utilized to graft host-guest molecules to capture antibiotics [17]. Surface functionalization of PP fibers with cold oxygen plasma is a well-known technique $[18,19]$. Cold oxygen plasma at low pressure has been especially considered as the most suitable plasma treatment, without changing their bulk properties, for PP fibers [20].

The new advancement in the preparation of medical devices is focused on producing biocompatible materials [21,22]. Thus, dopamine, a bio-inspired material, receives the attention of researchers due to the properties of self-polymerization to polydopamine (PDA) and co-deposition onto surfaces of the materials $[23,24]$. The PDA coating, or functionalization of material, is a straightforward approach by dipping materials in $\mathrm{pH}$ 8.5. The dopamine self-polymerization can be controlled by reaction time or concentration $[25,26]$.

In this work, we prepared antibiotic PP mesh materials functionalized with dopamine via cold $\mathrm{O}_{2}$ plasma treatment. PP mesh fiber surfaces were activated with oxygen plasma to create hydroxyl or carboxyl groups and then plasma treated meshes were further modified with self-polymerized dopamine for 12-24 h. The PDA incorporated PP meshes were soaked in levofloxacin $(0.5 \%)$ for $24 \mathrm{~h}$ to absorb water-soluble drugs for antimicrobial properties, as shown in Figure 1.

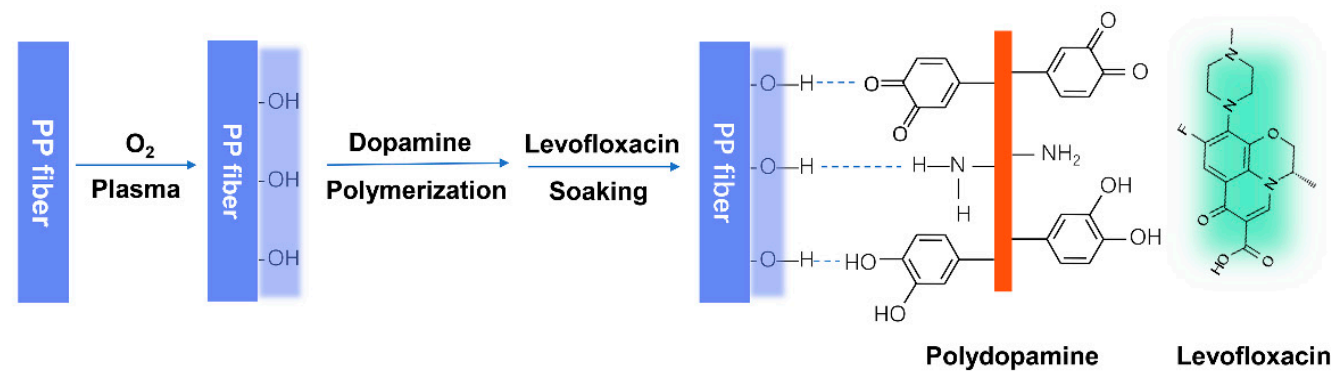

Figure 1. The schematic illustration of PDA coating and subsequent loading levofloxacin on PP meshes.

The PDA coated PP meshes were evaluated for surface morphology, structural changes, antimicrobial properties, and drug release. The results confirmed that dopamine was incorporated on PP fiber surfaces and formed strong interfacial bonding between PP meshes and PDA. Moreover, PDA coated and levofloxacin soaked meshes showed excellent antimicrobial inhibition zones and demonstrated a controlled drug release.

\section{Materials and Methods}

\subsection{Materials}

Light weight $\left(27 \mathrm{~g} / \mathrm{m}^{2}\right)$ and large hexagonal pore (sized $3.5 \mathrm{~mm} \times 2.5 \mathrm{~mm}$ ) monofilament polypropylene mesh devices were received from Nantong Newtec Textile and Chemical Fiber Co., Ltd., Nantong, China. Levofloxacin hydrochloride (98\%) was purchased from Energy Chemicals, Shanghai, China. Dopamine and tris(hydroxymethyl)aminomethane (Tris) were received from Aladdin Chemicals Ltd., Shanghai, China. 


\subsection{Surface Functionalization of PP with Oxygen Plasma}

PP mesh samples of equal size $(10 \mathrm{~cm} \times 10 \mathrm{~cm})$ were surface activated using an HD-300 cold plasma machine (Changzhou Zhongke Changtai Plasma Technology Co., Ltd., Changzhou, China). The samples were surface functionalized with oxygen plasma using optimized parameters of time $(300 \mathrm{~s})$, power $(45 \mathrm{~W})$, and pressure $(10 \mathrm{~Pa})$. However, flow of oxygen gas was less than 0.2 bars. PP samples after plasma treatment were named as PL-PP.

\subsection{Coating of Polydopamine (PDA) on PP Meshes}

Dopamine solution was prepared by dissolving $0.1 \mathrm{~g}$ of dopamine $(2 \mathrm{mg} / \mathrm{mL})$ in $50 \mathrm{~mL}$ of Tris-HCl buffer (10 mM, pH 8.5) [25]. The liquor ratio of the PP meshes to the solution was 1:100. Dopamine self-polymerization (non-plasma and oxygen plasma treated samples) was performed at a temperature $37^{\circ} \mathrm{C}$, with continuous stirring ( $80 \mathrm{rpm}$ ) for $12-24 \mathrm{~h}$. After $12-24 \mathrm{~h}$ samples were taken out, rinsed several times with hot $\left(50^{\circ} \mathrm{C}\right)$ and cold deionized water, and dried at $50^{\circ} \mathrm{C}$ in a vacuum oven. All samples were coated with the same concentration, the only difference was time. PDA coated samples after plasma treatment for 12 and $24 \mathrm{~h}$ were named PL-Dop-12 and PL-Dop-24, respectively. Non-plasma treated samples of coated PDA were named Dop-12 and Dop-24.

\subsection{Loading and Release of Levofloxacin}

The PDA coated PP $(0.5 \mathrm{~g})$ meshes were soaked for $24 \mathrm{~h}$ in a $50 \mathrm{~mL}$ solution containing $0.3 \mathrm{~g}(0.6 \%)$ levofloxacin. All samples were soaked in same concentration of levofloxacin for the same soaking time of $24 \mathrm{~h}$. After $24 \mathrm{~h}$, loading samples were taken out and dried at $40^{\circ} \mathrm{C}$. Thus, after levofloxacin, loaded samples were named Dop-LVFX-24, PL-Dop-LVFX-12, and PL-Dop-LVFX-24.

The drug release profiles of levofloxacin loaded samples $(2 \mathrm{~cm} \times 2 \mathrm{~cm})$ were measured in a centrifugal tube containing $8 \mathrm{~mL}$ of phosphate-buffered saline (PBS) and the PBS mixture with mesh samples was shaken the for required duration at $70 \mathrm{rpm}$. The UV-spectrophotometry (TU-1901 Beijing Purkinjie Co., Ltd., Beijing, China) was used at $37^{\circ} \mathrm{C}$ for absorption measurements. During each absorption measurement, $1 \mathrm{~mL}$ of the mixture solution of each sample was extracted and $1 \mathrm{~mL}$ of fresh PBS was added into the mixture. Absorption of all samples was measured at $290 \mathrm{~nm}$. The accumulative levofloxacin release (\%) was obtained as the ratio of levofloxacin release to the total drug loaded onto the samples. Average absorption values were used to calculate the levofloxacin release (\%).

\subsection{Characterization of Modified PP Meshes}

PDA coated and levofloxacin loaded samples were coated with platinum $(\mathrm{Pt})$ and observed with a Scanning Electron Microscope (SEM) (Quanta SEM 250, FEI ${ }^{\mathrm{TM}}$, Waltham, MA, USA). Moreover, an Energy Dispersive X-ray spectrometer (EDX) (ISIS 300, Oxford Instruments, Oxfordshire, UK) was connected with the SEM for element analysis of the PDA-modified and untreated PP samples. Fourier Transform Infrared Spectroscopy (FTIR) (Nicolet 6700, Thermo Fisher Scientific, Waltham, MA, USA) of Attenuated Total Reflection (ATR) mode was used to characterize all modified and control samples. All samples were analyzed in the wavenumber range of $500-4000 \mathrm{~cm}^{-1}$ with resolution of $4.0 \mathrm{~cm}^{-1}$. An atomic force microscope (AFM) (Technologies 5500, Keysight, Santa Rosa, CA, USA) was used to analyze the roughness of the different surfaces.

\subsection{Antibacterial Properties}

Qualitative Analysis

The agar diffusion test method was performed to assess the antibacterial properties of the dopamine coated and levofloxacin loaded samples described in our recently published paper in Polymers [27]. $400 \mu \mathrm{L}$ of $1 \times 10^{8} \mathrm{CFU} / \mathrm{mL}$ bacterial suspension was spread on agar plates. Sterilized swabs were used to evenly spread the bacteria. Two types of bacteria namely Escherichia (E.C) (ATCC ${ }^{\circledR}$ 
$25922^{\mathrm{TM}}$, Shanghai, China) and Staphylococcus aureus (S.A) (ATCC ${ }^{\circledR} 25923^{\mathrm{TM}}$, Shanghai, China) were used for antibacterial analysis of the modified meshes. Modified and untreated samples $(1 \mathrm{~cm} \times 1 \mathrm{~cm}$ square) were placed in the center of agar plates containing bacteria (S.A and E.C). The agars plated with samples were incubated in a standard oven, maintaining $37^{\circ} \mathrm{C}$ for $24 \mathrm{~h}$. The zone of inhibition of each sample was measured with a digital vernier caliper and all results from the inhibition zones were measured in 4 directions and reported as the average values. The zone of inhibition was measured using the following formula: $X=(Y-Z) / 2$, where $X=$ the inhibition zone, $Y=$ the inhibition zone measured after $24 \mathrm{~h}$ incubation, and $Z=$ the inhibition zone before incubation.

Furthermore, we observed the antibacterial properties of the modified and untreated PP samples in a controlled environment of $37^{\circ} \mathrm{C}$. After each day, samples were taken out of the oven and transferred to fresh agar plates containing bacteria. The zone of inhibition was measured to see the change in antibacterial properties according to the number of days.

\section{Results and Discussion}

\subsection{Polydopamine Coated and Levofloxacin Loaded}

Polypropylene (PP) warp knitted meshes were soaked in a weak alkaline solution of dopamine. It was expected that dopamine (PDA) would self-polymerize and co-deposit onto PP meshes. Two steps were performed in the surface modification of PP meshes. In the first step, PP meshes were activated with oxygen plasma and in the second step more PDA was adhered to oxygen plasma activated PP meshes via hydrogen bond.

Plasma treated and untreated PP meshes were coated in the same concentration of dopamine and at the same temperature $\left(37^{\circ} \mathrm{C}\right)$ for 12 and $24 \mathrm{~h}$ and we received a marginal difference between oxygen plasma treated and untreated coating amounts. The weight increase of oxygen plasma treated and untreated meshes, for 12 and $24 \mathrm{~h}$, is shown in Figure 2. The corresponding weight of the Dop-12 and Dop-24 is $0.3 \%$ and $0.5 \%$, respectively. However, the average weight increase of PL-Dop-12 and PL-Dop-24 is $2.81 \%$ and $3.75 \%$, respectively. Moreover, after 24 to $36 \mathrm{~h}$ soaking times there was no change in the amount of dopamine coating.

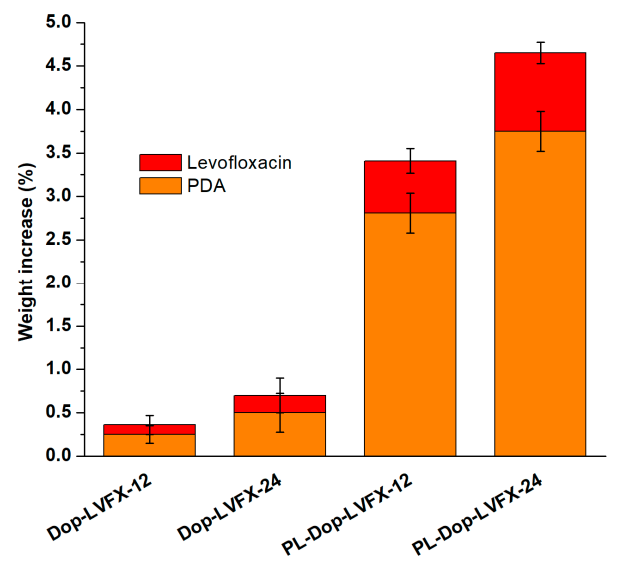

Figure 2. The weight increase (\%) of untreated and plasma treated PP meshes after PDA and levofloxacin coating.

All modified PP meshes were soaked in the same concentration $(0.6 \%)$ of levofloxacin for $24 \mathrm{~h}$. Plasma treated and PDA coated PP meshes were weighed before and after loading levofloxacin. From Figure 2, PL-Dop-LVFX-12 and PL-Dop-LVFX-24 increase the corresponding weights by $0.6 \%$ and $0.9 \%$, respectively. Moreover, samples without plasma treatment (Dop-LVFX-12 and Dop-LVFX-24) increase corresponding weight by only $0.2 \%$. Therefore, non-plasma treated samples (Dop-24, Dop-LVFX-24) and plasma treated samples (PL-Dop-LVFX-12 and PL-Dop-LVFX-24) were prepared for further characterization. 


\subsection{Surface Morphology of Modified PP Meshes}

Surface roughness of PP control and PL-PP meshes were observed using an AFM (Figure 3). Prior to oxygen plasma activation the surface of untreated mesh fibers is smooth with obvious mark lines. After oxygen plasma modification, the surface of PP fibers becomes rougher and the surface piles are more visible, instead of line marks. Moreover, after the oxygen plasma treatment, $S_{\mathrm{q}}$ and $S_{\mathrm{a}}$ parameters of the modified meshes are increased by $55.5 \%$ and $21.2 \%$, respectively, showing a rougher surface as compared to the PP control fibers. Therefore, oxygen plasma functionalization significantly changes the surface of PP meshes, which is consistent with the literature [28].

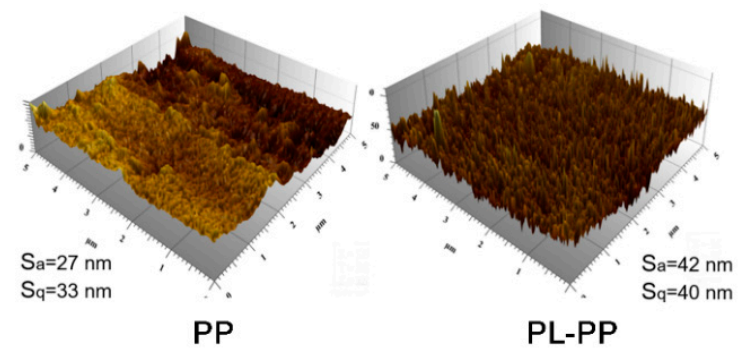

Figure 3. AFM of PP control and PL-PP.

Figure 4 shows SEM images of the modified PP samples. It can be observed that the PP control displays identical sharp and thin lines before oxygen plasma treatment (Figure 4a1,a2). Nevertheless, after plasma treatment a fuzzy and slightly dark appearance, without cut and lines (Figure 4b1,b2) shows a rougher surface of PP mesh. Moreover, for Dop-24 (Figure 4c1,c2), PDA can be observed on the surfaces of fibers with small beads, but PP mesh fibers are still not fully covered with PDA. On the other hand, PL-Dop-12 (Figure 4d1,d2) shows a more PDA coated area of PP fibers. This may be because, after plasma treatment, $-\mathrm{OH}$ groups on the PP surface enhanced the adherence of PDA more effectively via hydrogen bond, compared to non-plasma treated meshes. Moreover, PL-Dop-24 (Figure 4e1,e2) shows remarkably increased coating with wider beads, which covered whole spherical fiber area.
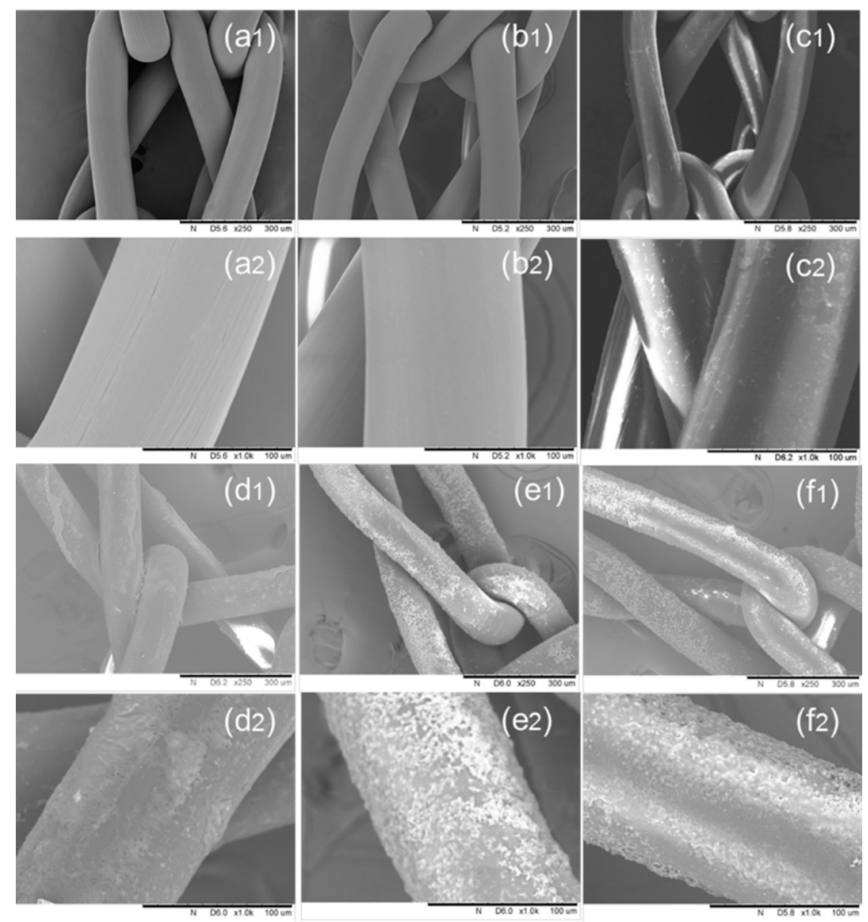

Figure 4. SEM images as follows: PP control (a1,a2), PL-PP (b1,b2), Dop-24 (c1,c2), PL-Dop-12 (d1,d2), PL-Dop-24 (e1,e2), and PL-Dop-LVFX-24 (f1,f2). Top row (a1-f1) $\times 250$ and bottom row $(\mathbf{a} 2-\mathbf{f} 2) \times 1000$. 
Furthermore, PL-Dop-LVFX-24 (Figure 4f1,f2) shows a more swollen and brighter structure, which may be due to levofloxacin absorbed by PDA coated meshes via catechol and hydrogen bond. Above all, plasma treated meshes could be effectively coated with PDA. This is due to the plasma surface modification of PP fibers, which increases surface wettability [29] as well as surface adhesion [30] for PDA coating.

\subsection{Chemical Characterization of Modified PP Meshes}

Figure 5 displays the element analysis of PP control and modified meshes using Energy Despersive EDX. Figure 5a shows only the carbon (C) atom peak within $0.5 \mathrm{keV}$, which confirms PP fibers. PL-PP meshes show additional oxygen atom $(\mathrm{O})$, which confirms oxygen-containing groups, generated on the surface of PP meshes after oxygen plasma treatment, as shown in Figure 5b. Furthermore, Figure $5 c$ displays carbon $(\mathrm{C})$, oxygen $(\mathrm{O})$, and an additional nitrogen $(\mathrm{N})$ atom between the oxygen and carbon peaks, which confirms that the PP meshes were coated by PDA and that the oxygen atoms' weight (\%) increased from $2.85 \%$ to $7.89 \%$. Moreover, after levofloxacin loading, the presence of fluorine $(\mathrm{F})$ atom is displayed within $0.6 \mathrm{keV}$ (Figure $5 \mathrm{~d}$ ). Thus, it proves the existence of levofloxacin on the surface of PDA coated PP meshes. These results are similar to our recent published paper, except for a different weight (\%) of each element [27].

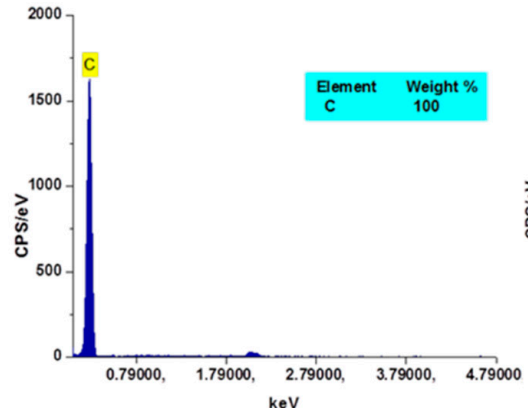

(a)

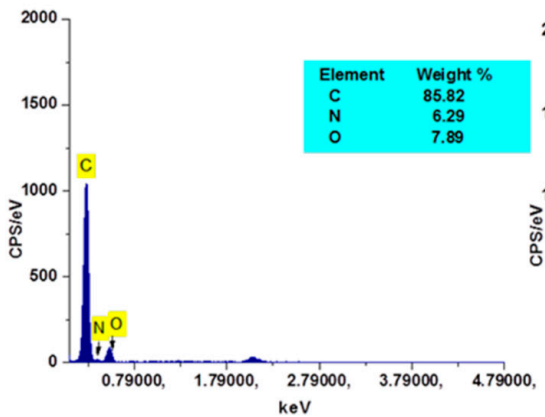

(C)

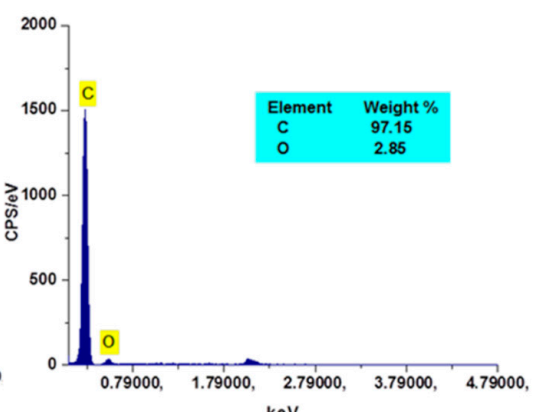

(b)

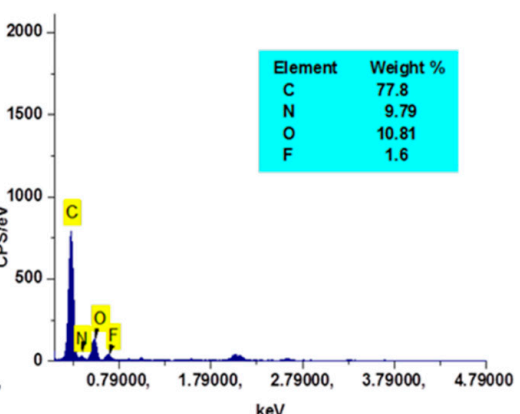

(d)

Figure 5. EDX spectra and weight of element (\%) (a) PP control; (b) PL-PP; (c) PL-Dop-24; and (d) PL-Dop-LVFX-24.

FTIR spectra of untreated and treated samples are shown in Figure 6. The PP control shows peaks at 2950, 2916, 1452, and $1376 \mathrm{~cm}^{-1}$ [17]. Thus, except for the PP control, PL-PP meshes display additional peaks at $3264 \mathrm{~cm}^{-1}$, which is due to the hydroxyl $(-\mathrm{OH})$ on the surface of PP meshes. Moreover, it can be observed that Dop-24 shows slight vibration peaks at 1626 and $1532 \mathrm{~cm}^{-1}$, which is due to the establishment of $v \mathrm{C}=\mathrm{O}$ and $\delta \mathrm{NH}$, respectively. Furthermore, $\mathrm{C}-\mathrm{H}(\nu \mathrm{CH})$ stretch can be observed at $1290 \mathrm{~cm}^{-1}$. PL-Dop-24 shows similar peaks of 1626 and $1532 \mathrm{~cm}^{-1}$. More intensity of the absorbance band is found, which shows hydrogen bonding between PDA and PP meshes. Moreover, PL-Dop-LVFX-24 shows similar peaks as PDA coating peaks because levofloxacin was just loaded on the layer and did not react with PDA. 


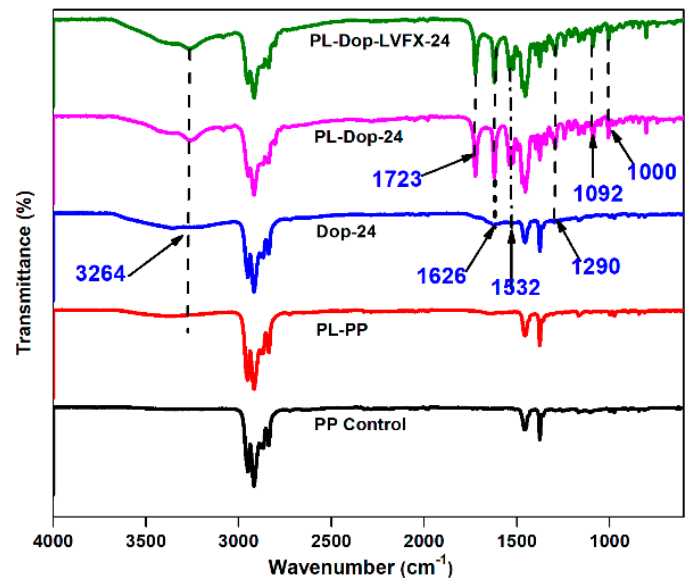

Figure 6. FTIR spectra (ATR) of PP control, PL-PP, Dop-24, PL-Dop-24, and PL-Dop-LVFX-24.

\subsection{Antibacterial Activity}

Antibacterial activity of PP meshes at different stages of modification was performed by the agar diffusion method, as shown in Figure 7. It can be observed that the PP control did not display antibacterial properties. Nevertheless, PP meshes activated with oxygen plasma have slight antibacterial properties. Dop-LVFX-24 showed good antibacterial properties. The average inhibition zone for E.C and S.A was 9.6 and $8.5 \mathrm{~mm}$, respectively. With plasma treatment, PL-Dop-LVFX-12 performed better and showed an average inhibition zone of 12.5 and $12 \mathrm{~mm}$ for E.C and S.A, respectively. PL-Dop-LVFX-24 demonstrated excellent antibacterial properties with an inhibition zone of 15 and $14.5 \mathrm{~mm}$ for E.C and S.A, respectively.

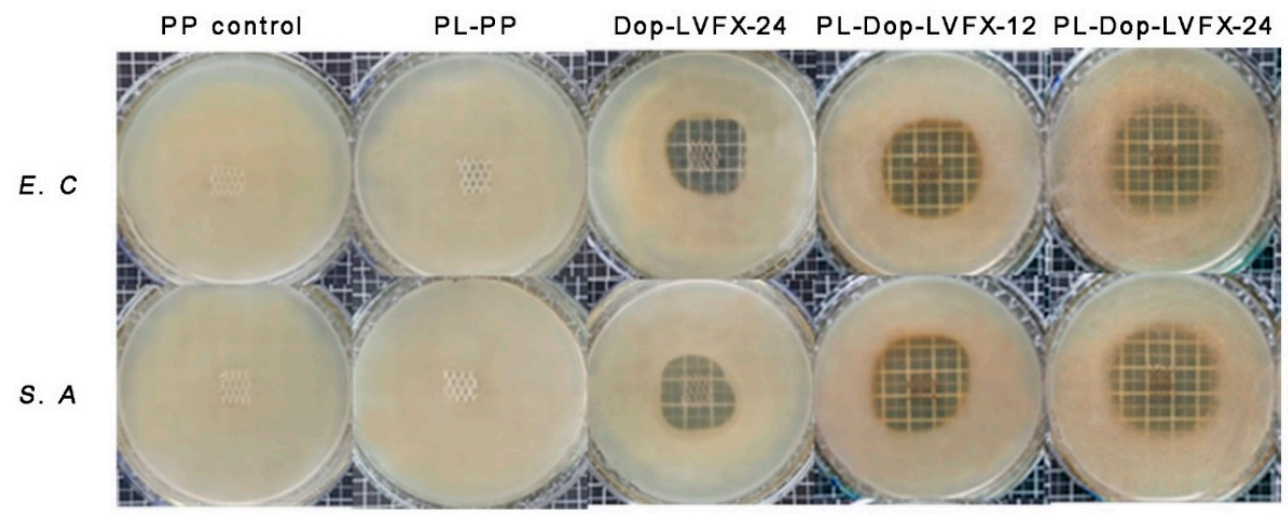

Figure 7. Inhibition zone diameter of PP control, PL-PP, Dop-LVFX-24, PL-Dop-LVFX-12, and PL-Dop-LVFX-24. All samples in the top row = E.C and the bottom row $=$ S.A.

Moreover, antibacterial release properties of modified PP meshes (Figure 8) were determined after each $24 \mathrm{~h}$. It can be observed that Dop-LVFX-24 exhibited antibacterial properties for only two days, as shown in Figure 8a. This may because less levofloxacin was adhered on the surface due to less PDA coating. However, PDA coated PP meshes, after plasma treatment for $12 \mathrm{~h}$ and soaked in the levofloxacin (Dop-LVFX-12), showed good antibacterial properties for 5 days, with a reduced regular inhibition zone throughout 5 days. The minimum inhibition zone on 5th day was 3 and $2.5 \mathrm{~mm}$ for E.C and S.A, respectively (Figure 8 b). A bigger average inhibition zone was displayed by samples using E.C during the entire 5 days. Moreover, PDA coated meshes, for $24 \mathrm{~h}$ and soaked in levofloxacin (Dop-LVFX-24), demonstrated a more sustained antibacterial release for 6 days (Figure 8c). It was also observed that the inhibition zone of $E . C$ was greater, in comparison to $S . A$, for an entire 6 days, but on 4th day almost the same inhibition zone was shown for the two types of bacteria. The minimum inhibition zone for E.C and S.A was 2.99 and $2.05 \mathrm{~mm}$ respectively. 


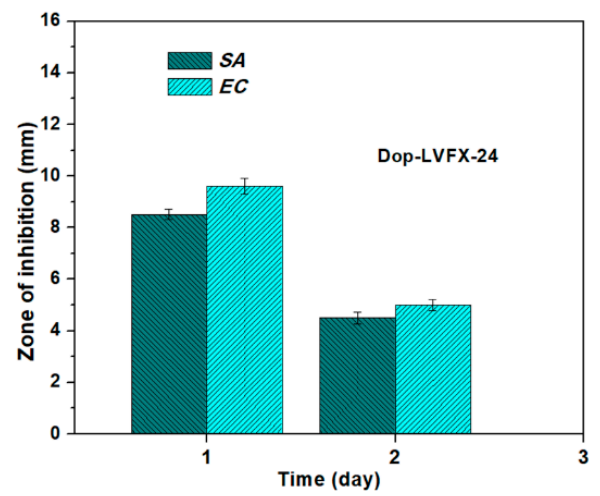

(a)

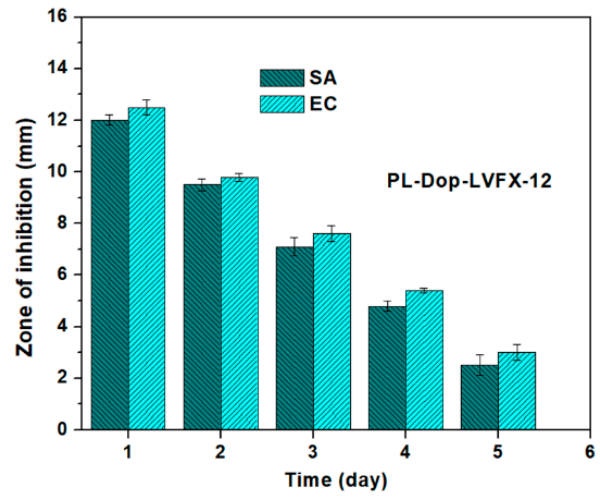

(b)

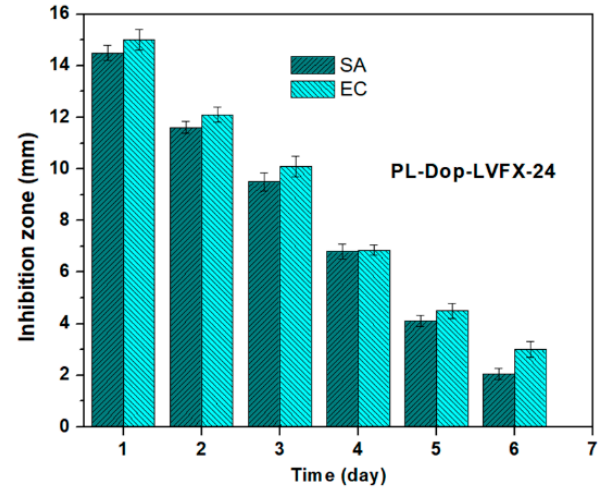

(c)

Figure 8. Antibacterial release properties of (a) Dop-LVFX-24, (b) PL-Dop-LVFX-12, and (c) PL-Dop-LVFX-24.

In our previous work, we prepared PP meshes with a one-step coating of PDA, without plasma treatment [31]. Herein, we found better results with PDA coating using oxygen plasma. Thus, we compared antibacterial performance of PDA coating without plasma and PDA coating with oxygen plasma. Overall, plasma treated and PDA coated PP meshes, PL-Dop-LVFX-12 and PL-Dop-LVFX-24, displayed more sustained antibacterial properties than non-plasma treated PP meshes (Dop-LVFX-24). This may because plasma treatment improved the coating efficiency of PDA onto PP fibers with increased corresponding weight and strong bonding, which may hold more levofloxacin and release antibacterial properties for a longer duration of time compared to non-plasma treated PP meshes.

\subsection{Drug Release Profile of PDA Coated and Levofloxacin Loaded PP Meshes}

The average accumulative drug release (\%) of three samples (Dop-LVFX-24, PL-Dop-LVFX-12, and PL-Dop-LVFX-24) was performed in a neutral environment using a PBS solution. From Figure 9 and Table 1, it can be observed that Dop-LVFX-24 showed fast drug release and about $100 \%$ of the drug was released within first $6 \mathrm{~h}$, though a major portion $(80 \%)$ of the drug was released within $3 \mathrm{~h}$. Moreover, PL-Dop-LVFX-12 displayed a sustained drug release profile. In first $3 \mathrm{~h}, 65 \%$ of drug was released; after $6 \mathrm{~h}$, the accumulative drug release was $78.8 \%$; and after $12 \mathrm{~h}, 91.9 \%$ was released. Further sustained drug release $(99.1 \%)$ continued up to $24 \mathrm{~h}$. Moreover, PL-Dop-LVFX-24 displayed more sustained levofloxacin release compared to PL-Dop-LVFX-12. In the first $3 \mathrm{~h}$, an accumulative $60.88 \%$ of the drug was released. After 6 h, $72.2 \%$ of levofloxacin was released. At 12 h, $86.3 \%$ of the drug was released and a total of $93.3 \%$ of drug was released at $24 \mathrm{~h}$. Thus, PL-Dop-LVFX-24 samples showed sustained accumulative drug release. Drug releases of all three samples can explain antibacterial activity performance. The PL-Dop-LVFX-24 sample could be a suitable product and may be used for hernia mesh implantation. 


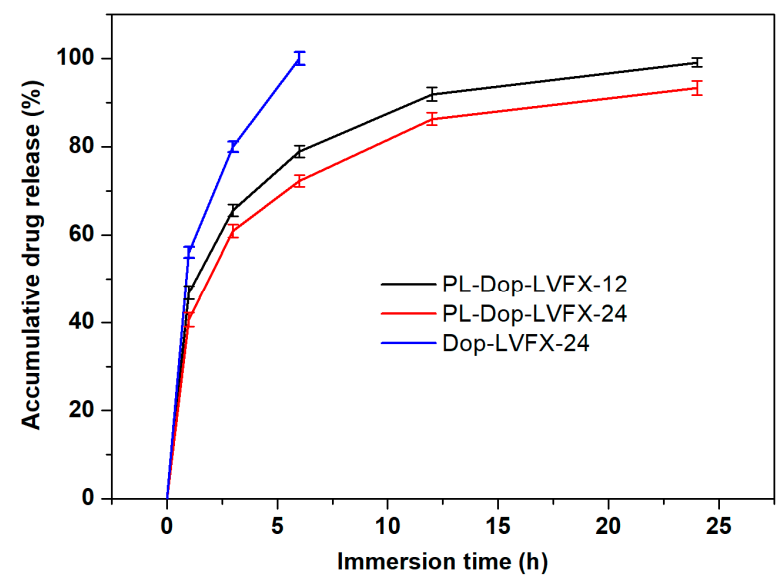

Figure 9. Drug release curves of PDA coated and levofloxacin loaded PP meshes.

Table 1. The average accumulative drug release of three samples.

\begin{tabular}{cccc}
\hline Time (h) & Dop-LVFX-24 (\%) & PL-Dop-LVFX-12 (\%) & PL-Dop-LVFX-24 (\%) \\
\hline $\mathbf{0}$ & 0 & 0 & 0 \\
$\mathbf{1}$ & $56 \pm 1.3$ & $47 \pm 1.5$ & $41 \pm 1.6$ \\
$\mathbf{3}$ & $80 \pm 1.2$ & $65 \pm 1.3$ & $61 \pm 1.5$ \\
$\mathbf{6}$ & $100 \pm 1.5$ & $79 \pm 1.3$ & $72 \pm 1.3$ \\
$\mathbf{1 2}$ & - & $92 \pm 1.5$ & $86 \pm 1.4$ \\
$\mathbf{2 4}$ & - & $99 \pm 1.0$ & $93 \pm 1.6$ \\
\hline
\end{tabular}

In our previous study, cyclodextrins were used to capture levofloxacin HCL for antibacterial and drug release properties. The release of the drug was observed for at least $24 \mathrm{~h}$ [32]. Herein, polydopamine (PDA) coated PP meshes were soaked in the levofloxacin $\mathrm{HCl}$ solution and modified PP meshes showed a better antibacterial inhibition zone than PP meshes modified with cyclodextrins, but cyclodextrins had released the drug for a longer duration of time than polydopamine. The reason for this could be that cyclodextrins have cavities which releases the drug slowly.

Moreover, commonly used drug carriers, such as cyclodextrins [33-35] and chitosan [36,37], may release drugs for longer durations of time, compared to polydopamine, but the inhibition zones may be smaller. Overall, PDA modified PP meshes released levofloxacin HCL for $24 \mathrm{~h}$ and showed excellent antibacterial inhibition zones of 15 and $14.5 \mathrm{~mm}$ for E.C and S.A, respectively. Considering the burst release of drugs, a hernia mesh infection is necessary to control in the initial stages of mesh implantation [32,38]. Thus, the PDA and levofloxacin HCL coated PP meshes with good drug release properties could be useful for prevention of hernia mesh infections.

\section{Conclusions}

Bio-inspired PDA coated antimicrobial PP meshes for hernia repair were successfully prepared. The oxygen plasma treated PP meshes show better results of PDA coating than the raw one. It is proved that PDA coated PP meshes, after oxygen plasma treatment, can absorb more levofloxacin and release the drug for a longer suitable time, which demonstrates reasonable antimicrobial release properties. Thus, plasma treated PP meshes coated with PDA and loaded with levofloxacin may be a good choice for prevention of hernia mesh infections.

Author Contributions: Conceptualization X.S., N.S. and Y.L.; Methodology X.S. and Y.Q.; Software, N.S.; Validation, A.K. and X.S.; Writing Original Draft, L.W. and J.G.; Writing-Review and Editing, G.B. and R.G.

Funding: The Fundamental Research Funds for the Central Universities (No. 17D110111 \& 2232018G-01), the National Key Research Development Program of China (No. 2016YFB 0303300), and 111 project "Biomedical Textile Material Science and Technology" (No. B07024). 
Acknowledgments: Thanks to students of the Biomedical Textile Materials Group for their help during experiments and testing.

Conflicts of Interest: The authors declare no conflict of interest.

\section{References}

1. Guillaume, O.; Perez-Tanoira, R.; Fortelny, R.; Redl, H.; Moriarty, T.F.; Richards, R.G.; Eglin, D.; Petter Puchner, A. Infections associated with mesh repairs of abdominal wall hernias: Are antimicrobial biomaterials the longed-for solution? Biomaterials 2018, 167, 15-31. [CrossRef] [PubMed]

2. Greca, F.; Paula, J.; Biondo-Simões, M.; Costa, F.; Silva, A.; Time, S.; Mansur, A. The influence of differing pore sizes on the biocompatibility of two polypropylene meshes in the repair of abdominal defects. Hernia 2001, 5, 59-64. [PubMed]

3. Hazebroek, E.J.; Ng, A.; Yong, D.H.; Berry, H.; Leibman, S.; Smith, G.S. Evaluation of lightweight titanium-coated polypropylene mesh (TiMesh) for laparoscopic repair of large hiatal hernias. Surg. Endosc. 2008, 22, 2428-2432. [CrossRef] [PubMed]

4. Jerabek, J.; Novotny, T.; Vesely, K.; Cagas, J.; Jedlicka, V.; Vlcek, P.; Capov, I. Evaluation of three purely polypropylene meshes of different pore sizes in an onlay position in a New Zealand white rabbit model. Hernia 2014, 18, 855-864. [CrossRef] [PubMed]

5. Kulaga, E.; Ploux, L.; Balan, L.; Schrodj, G.; Roucoules, V. Mechanically responsive antibacterial plasma polymer coatings for textile biomaterials. Plasma Process. Polym. 2014, 11, 63-79. [CrossRef]

6. Knetsch, M.L.W.; Koole, L.H. New strategies in the development of antimicrobial coatings: The example of increasing usage of silver and silver nanoparticles. Polymers 2011, 3, 340-366. [CrossRef]

7. Mazaki, T.; Mado, K.; Masuda, H.; Shiono, M.; Tochikura, N.; Kaburagi, M. A randomized trial of antibiotic prophylaxis for the prevention of surgical site infection after open mesh-plug hernia repair. Am. J. Surg. 2014, 207, 476-484. [CrossRef] [PubMed]

8. Mazaki, T.; Mado, K.; Masuda, H.; Shiono, M. Antibiotic prophylaxis for the prevention of surgical site infection after tension-free hernia repair: A Bayesian and frequentist meta-analysis. J. Am. Coll. Surg. 2013, 217, 788-801. [CrossRef] [PubMed]

9. Perez-Kohler, B.; Fernandez-Gutierrez, M.; Pascual, G.; Garcia-Moreno, F.; San Roman, J.; Bellon, J.M. In vitro assessment of an antibacterial quaternary ammonium-based polymer loaded with chlorhexidine for the coating of polypropylene prosthetic meshes. Hernia 2016, 20, 869-878. [CrossRef] [PubMed]

10. Zhang, Z.; Tang, J.; Wang, H.; Xia, Q.; Xu, S.; Han, C.C. Controlled antibiotics release system through simple blended electrospun fibers for sustained antibacterial effects. ACS Appl. Mater. Interfaces 2015, 7, 26400-26404. [CrossRef] [PubMed]

11. Sanbhal, N.; Miao, L.; Xu, R.; Khatri, A.; Wang, L. Physical structure and mechanical properties of knitted hernia mesh materials: A review. J. Ind. Text. 2017, 48, 333-360. [CrossRef]

12. Muzio, G.; Miola, M.; Perero, S.; Oraldi, M.; Maggiora, M.; Ferraris, S.; Vernè, E.; Festa, V.; Festa, F.; Canuto, R.A.; et al. Polypropylene prostheses coated with silver nanoclusters/silica coating obtained by sputtering: Biocompatibility and antibacterial properties. Surf. Coat. Technol. 2017, 319, 326-334. [CrossRef]

13. Labay, C.; Canal, J.M.; Modic, M.; Cvelbar, U.; Quiles, M.; Armengol, M.; Arbos, M.A.; Gil, F.J.; Canal, C. Antibiotic-loaded polypropylene surgical meshes with suitable biological behaviour by plasma functionalization and polymerization. Biomaterials 2015, 71, 132-144. [CrossRef] [PubMed]

14. Nisticò, R.; Rosellini, A.; Rivolo, P.; Faga, M.G.; Lamberti, R.; Martorana, S.; Castellino, M.; Virga, A.; Mandracci, P.; Malandrino, M.; et al. Surface functionalisation of polypropylene hernia-repair meshes by RF-activated plasma polymerisation of acrylic acid and silver nanoparticles. Appl. Surf. Sci. 2015, 328, 287-295. [CrossRef]

15. Nisticò, R.; Magnacca, G.; Faga, M.G.; Gautier, G.; D’Angelo, D.; Ciancio, E.; Lamberti, R.; Martorana, S. Effect of atmospheric oxidative plasma treatments on polypropylenic fibers surface: Characterization and reaction mechanisms. Appl. Surf. Sci. 2013, 279, 285-292. [CrossRef]

16. Zhang, Z.; Zhang, T.; Li, J.; Ji, Z.; Zhou, H.; Zhou, X.; Gu, N. Preparation of poly(L-lactic acid)-modified polypropylene mesh and its antiadhesion in experimental abdominal wall defect repair. J. Biomed. Mater. Res. Part. B Appl. Biomater. 2014, 102, 12-21. [CrossRef] [PubMed] 
17. Sanbhal, N.; Mao, Y.; Sun, G.; Xu, R.F.; Zhang, Q.; Wang, L. Surface modification of polypropylene mesh devices with cyclodextrin via cold plasma for hernia repair: Characterization and antibacterial properties. Appl. Surf. Sci. 2018, 439, 749-759. [CrossRef]

18. Jelil, R.A. A review of low-temperature plasma treatment of textile materials. J. Mater. Sci. 2015, 50, 5913-5943. [CrossRef]

19. Lai, J.; Sunderland, B.; Xue, J.; Yan, S.; Zhao, W.; Folkard, M.; Michael, B.D.; Wang, Y. Study on hydrophilicity of polymer surfaces improved by plasma treatment. Appl. Surf. Sci. 2006, 252, 3375-3379. [CrossRef]

20. Khelifa, F.; Ershov, S.; Habibi, Y.; Snyders, R.; Dubois, P. Free-radical-induced grafting from plasma polymer surfaces. Chem. Rev. 2016, 116, 3975-4005. [CrossRef] [PubMed]

21. Patel, H.; Ostergard, D.R.; Sternschuss, G. Polypropylene mesh and the host response. Int. Urogynecol. J. 2012, 23, 669-679. [CrossRef] [PubMed]

22. Perez-Kohler, B.; Bayon, Y.; Bellon, J.M. Mesh infection and hernia repair: A review. Surg. Infect. 2016, 17, 124-137. [CrossRef] [PubMed]

23. Liu, Y.; Fang, Y.; Qian, J.; Liu, Z.; Yang, B.; Wang, X. Bio-inspired polydopamine functionalization of carbon fiber for improving the interfacial adhesion of polypropylene composites. RSC Adv. 2015, 5, 107652-107661. [CrossRef]

24. Ngo, T.H.A.; Nguyen, D.T.; Do, K.D.; Nguyen, T.T.M.; Mori, S.; Tran, D.T. Surface modification of polyamide thin film composite membrane by coating of titanium dioxide nanoparticles. J. Sci. Adv. Mater. Devices 2016, 1, 468-475. [CrossRef]

25. Ying, T.; Guo-Xin, T.; Cheng-Yun, N.; Xi-Cang, R.; Yu, Z.; Lei, Z. Bioinspired polydopamine functionalization of titanium surface for silvernanoparticles immobilization with antibacterial property. J. Inorg. Mater. 2014, 29, 1320-1326. [CrossRef]

26. Zhang, R.X.; Breaken, L.; Liu, T.Y.; Luis, P.; Wang, X.L.; Van der Bruggen, B. Remarkable anti-fouling performance of $\mathrm{TiO}_{2}$-modified TFC membranes with mussel-inspired polydopamine binding. Appl. Sci. 2017, 7, 81. [CrossRef]

27. Sanbhal, N.; Mao, Y.; Sun, G.; Li, Y.; Peerzada, M.; Wang, L. Preparation and characterization of antibacterial polypropylene meshes with covalently incorporated $\beta$-cyclodextrins and captured antimicrobial agent for Hernia repair. Polymers 2018, 10, 58. [CrossRef]

28. Vishnuvarthanan, M.; Rajeswari, N. Effect of mechanical, barrier and adhesion properties on oxygen plasma surface modified PP. Innov. Food Sci. Emerg. Technol. 2015, 30, 119-126. [CrossRef]

29. Fauland, G.; Constantin, F.; Gaffar, H.; Bechtold, T. Production scale plasma modification of polypropylene baselayer for improved water management properties. J. Appl. Polym. Sci. 2015, 132, 41294. [CrossRef]

30. Sorrentino, L.; Carrino, L.; Napolitano, G. Oxygen cold plasma treatment on polypropylene: Influence of process parameters on surface wettability. Surf. Eng. 2013, 23, 247-252. [CrossRef]

31. Sanbhal, N.; Saitter, X.; Peerzada, M.; Habboush, A.; Wang, F.; Wang, L. One-step surface functionalized hydrophilic polypropylene meshes for hernia repair using bio-inspired polydopamine. Fibres 2019, 7, 6. [CrossRef]

32. Sanbhal, N.; Saitaer, X.; Li, Y.; Mao, Y.; Zou, T.; Sun, G.; Wang, L. Controlled levofloxacin release and antibacterial properties of $\beta$-cyclodextrins-grafted polypropylene mesh devices for hernia repair. Polymers 2018, 10, 493. [CrossRef]

33. Laurent, T.; Kacem, I.; Blanchemain, N.; Cazaux, F.; Neut, C.; Hildebrand, H.F.; Martel, B. Cyclodextrin and maltodextrin finishing of a polypropylene abdominal wall implant for the prolonged delivery of ciprofloxacin. Acta Biomater. 2011, 7, 3141-3149. [CrossRef] [PubMed]

34. Otero-Espinar, F.J.; Torres-Labandeira, J.J.; Alvarez-Lorenzo, C.; Blanco-Méndez, J. Cyclodextrins in drug delivery systems. J. Drug Deliv. Sci. Technol. 2010, 20, 289-301. [CrossRef]

35. Thatiparti, T.R.; Shoffstall, A.J.; von Recum, H.A. Cyclodextrin-based device coatings for affinity-based release of antibiotics. Biomaterials 2010, 31, 2335-2347. [CrossRef] [PubMed]

36. Avetta, P.; Nisticò, R.; Faga, M.G.; D’Angelo, D.; Boot, E.A.; Lamberti, R.; Martorana, S.; Calza, P.; Fabbri, D.; Magnacca, G. Hernia-repair prosthetic devices functionalised with chitosan and ciprofloxacin coating: Controlled release and antibacterial activity. J. Mater. Chem. B 2014, 2, 5287-5294. [CrossRef] 
37. Nisticò, R.; Faga, M.G.; Gautier, G.; Magnacca, G.; D’Angelo, D.; Ciancio, E.; Piacenza, G.; Lamberti, R.; Martorana, S. Physico-chemical characterization of functionalized polypropylenic fibers for prosthetic applications. Appl. Surf. Sci. 2012, 258, 7889-7896. [CrossRef]

38. Erdas, E.; Medas, F.; Pisano, G.; Nicolosi, A.; Calo, P.G. Antibiotic prophylaxis for open mesh repair of groin hernia: Systematic review and meta-analysis. Hernia 2016, 20, 765-776. [CrossRef] [PubMed]

(C) 2019 by the authors. Licensee MDPI, Basel, Switzerland. This article is an open access article distributed under the terms and conditions of the Creative Commons Attribution (CC BY) license (http:/ / creativecommons.org/licenses/by/4.0/). 